\title{
Epidemiological trends of Reported Legionnaires' disease in Houston, Texas, 2014-2017
}

\section{Razina Khayat, Najmus Abdullah, Sudipa Biswas, Hafeez Rehman, Kirstin Short}

Informatics, City of Houston, Houston, Texas, United States

Objective

To study trends and patterns in legionnaires' disease cases in Houston, Texas, from 2014-2017.

Introduction

Legionellosis is a respiratory illness that is mostly (80-90\%) caused by the bacterium Legionella pneumophila. It is associated with a mild febrile illness, Pontiac fever, or Legionnaires' disease [1], a source of severe, community-acquired pneumonia. Legionella bacteria mostly affect elderly persons specifically those with underlying debilitating illnesses and with lowered immune systems. Water is the major natural reservoir for Legionella, and the pathogen is found in many different natural and artificial aquatic environments such as cooling towers or water systems in buildings, including hospitals. An abrupt increase in the incidence of Legionnaires' has been noted since 2003 throughout the nation. According to CDC, about 6,000 cases of Legionnaires' disease were reported in the United State in 2015 [1]. Incidence rates of Legionnaires for the year 2015 were 1.06 and 1.90 (ref) for Texas and the United States respectively [2]. Increased number of reported cases might be due to the fact of an older population, more at risk individuals, aging plumbing infrastructure, and increased testing for Legionnaires' disease by various hospitals and laboratories.

\section{Methods}

Data were extracted from Houston's Electronic Disease Surveillance System (HEDSS) from January 1, 2014, to December 07, 2018. Confirmed cases were analyzed to examine the epidemiologic trends across years 2014 to 2018. Demographic characteristics such as age, race, and gender were also analyzed. Incidence rates, case fatality and time lapse from date of diagnosis to date of reporting to the health department were also studied. Data were analyzed using SAS statistical software, version 9.4. Only Houston residents were included in the analysis. To be considered confirmed, a case must be clinically compatible and fulfill at least one of the confirmatory laboratory criteria.

\section{Results}

There were 218 cases of LD reported to the City of Huston from 2014 to 2018 . Only 116 cases (53\%) were classified as confirmed. Reported cases may have been not confirmed due to the lack of fulfilling the case criteria for the case. Providers may have ordered a non-confirmative test, or the case may not have satisfied the clinical compatibility due to loss to follow-up or for other reasons.

Most of the confirmed cases were reported from larger for-profit hospitals (500+beds) in the area. The majority of cases were diagnosed by urinary antigen test $(95,82 \%)$. There were four deaths due to legionnaires disease during this period giving a case fatality rate of $3.4 \%$. Death rates were inaccurate, though, and could be higher than reported since cases were not followed up after being reported to the state. From 2014 to 2018, legionnaires' disease incidence rates increased from 0.71 to 1.36 per 100,000 , an average annual increase of $17 \%$.

In 2014-2018, the incidence of LD was higher among men compared with women. 67 cases (58\%) were male, and 49 (42\%) were female. Female cases remained stable throughout the years while male cases increased from 6 to 23, an increase of approximately four folds. The median age was 60 years with a range of 21 to 96 years. LD incidence increased with age; it was highest among residents 65 years and older (42,36\%). African Americans had the highest incidence of LD (40, 35\%) followed by Hispanics (29, $25 \%$ ). African Americans cases had more than doubled through years 2014-2018 from 6 to 13. Cases were higher in warmer months specifically in July (14) an August (13). 
ISDS 2019 Conference Abstracts

\section{Conclusions}

Cases were higher in the warmer months and the highest among the elderly, men, and those of African American race. ELR was the prime source of initial case reporting to the health department. The number of legionnaire's cases observed were increasing with each passing year. The ratio of confirmed cases to those reported were only $53 \%$ thus raising awareness and appropriate education to the investigators and providers are highly advised. It is critical to the control of LD that enhanced surveillance is maintained at a high level. Consequently, more consideration should be given for the more widespread use of Legionnaires confirming test when a patient presents with pneumonia.

Hospitals and other healthcare facilities often have large, complex water systems, making them potentially high-risk settings for transmission of legionellosis to vulnerable patients or residents. We recommend all healthcare facilities have a water management program to control Legionella.

\section{Acknowledgement}

We thank the flollowing agencies for providing data for this study:

1. Texas Department of State Health Services.

2. Houston Health Department, Diivision of Disease Prevention and Control.

3. Houston Electronic Disease Surveillance System.

\section{References}

1. Centers for Disease Control and Prevention. (2018). Infection Control Assessment Tools. Retrieved October 5, 2018, from https://www.cdc.gov/legionella/

2. Texas Health and Human Services. (2018). Legionellosis. Retrieved October,5, 2018, from https://www.dshs.texas.gov/idcu/disease/legionnaires/ 